\title{
Improved isotope-shift-based bounds on bosons beyond the Standard Model through measurements of the ${ }^{2} \mathbf{D}_{3 / 2}-{ }^{2} \mathbf{D}_{5 / 2}$ interval in $\mathbf{C a}^{+}$
}

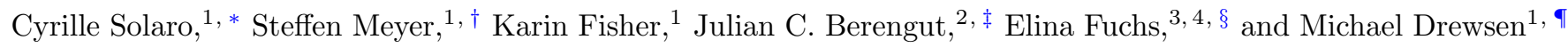 \\ ${ }^{1}$ Department of Physics and Astronomy, Aarhus University, DK-8000 Aarhus C, Denmark \\ ${ }^{2}$ School of Physics, University of New South Wales, Sydney NSW 2052, Australia \\ ${ }^{3}$ Fermilab, Theory Department, Batavia, IL 60510, USA \\ ${ }^{4}$ University of Chicago, Department of Physics, Chicago, IL 60637, USA
}

(Dated: May 4, 2020)

\begin{abstract}
We perform high-resolution spectroscopy of the $3 \mathrm{~d}^{2} \mathrm{D}_{3 / 2}-3 \mathrm{~d}{ }^{2} \mathrm{D}_{5 / 2}$ interval in all stable even isotopes of ${ }^{A} \mathrm{Ca}^{+}(\mathrm{A}=40,42,44,46$ and 48$)$ with an accuracy of $\sim 20 \mathrm{~Hz}$ using direct frequencycomb Raman spectroscopy. Combining these data with isotope shift measurements of the $4 \mathrm{~s}^{2} \mathrm{~S}_{1 / 2} \leftrightarrow$ $3 \mathrm{~d}{ }^{2} \mathrm{D}_{5 / 2}$ transition, we carry out a King plot analysis with unprecedented sensitivity to coupling between electrons and neutrons by bosons beyond the Standard Model. Furthermore, we estimate the sensitivity to such bosons from equivalent spectroscopy in $\mathrm{Ba}^{+}$and $\mathrm{Yb}^{+}$. Finally, the data yield isotope shifts of the $4 \mathrm{~s}^{2} \mathrm{~S}_{1 / 2} \leftrightarrow 3 \mathrm{~d}^{2} \mathrm{D}_{3 / 2}$ transition at 10 part-per-billion through combination with recent data of Knollmann et al. [1].
\end{abstract}

The Standard Model of particle physics (SM) cannot be complete since, e.g., it lacks a Dark Matter candidate, cannot produce the observed matter-antimatter asymmetry of the universe and does not explain the hierarchy between the Higgs mass and the Planck scale. Because the masses of new particles are unknown, searches for New Physics (NP) beyond the SM involve multiple frontiers (see e.g. Refs. [2-4] and references therein) ranging from high-energy colliders, high-intensity beam dumps, astrophysical and cosmological observations to high-precision table-top experiments. In the search for new long-range interactions, high-resolution spectroscopy of atoms and molecules is a driving force [5]. A recent example is to probe the existence of new bosons that couple to both nucleons and electrons from precisely measured isotope shifts. Conversely, agreement between the prediction based on the SM and experiments within their uncertainties allows for placing bounds on the coupling strength of the potential new interaction depending on the mass of the new boson. Except for few-electron systems [6], the main limitation in translating the experimental accuracy to a stringent bound is the theory uncertainty. To mitigate this problem, Delaunay et al. [7] proposed to measure isotope shifts of two different transitions of the same element and to look for a non-linearity of the so-called King Plot [8]. This allows to place bounds on long-range mediators [9], and thus to test various particle physics models [10]. For instance, the protophobic model $[11,12]$ of a new boson at $17 \mathrm{MeV} / c^{2}$ for the Be anomaly [13] is in reach of near-future $\mathrm{Sr} / \mathrm{Sr}^{+}$and $\mathrm{Yb}^{+}$King plot analyses $[9,10]$. This data-driven method requires only theory input for the new interaction, but is independent

\footnotetext{
* solaro@phys.au.dk

† steffen.meyer@phys.au.dk

‡ julian.berengut@unsw.edu.au

$\S$ elinafuchs@uchicago.edu

『 drewsen@phys.au.dk
}

of SM multi-electron and nuclear calculations - unless a non-linearity from higher-order SM effects is predicted at the level of experimental precision. After subtracting the predicted SM non-linearity, the residual non-linearity can be used to constrain a NP contribution. A King plot, however, requires at least four isotopes (preferably with zero nuclear spin) in order to test the linearity of the isotope shifts of the resulting three independent isotope pairs. Calcium is in this respect a good candidate with the five stable, spin-0 isotopes $\mathrm{A}=40,42,44,46$ and 48. Previously, Gebert et al. reported measurements of two dipole allowed transitions, $4 \mathrm{~s}^{2} \mathrm{~S}_{1 / 2} \leftrightarrow 4 \mathrm{p}{ }^{2} \mathrm{P}_{1 / 2}$ (397-nm) and $3 \mathrm{~d}^{2} \mathrm{D}_{3 / 2} \leftrightarrow 4 \mathrm{p}{ }^{2} \mathrm{P}_{1 / 2}$ (866-nm), in the four ${ }^{40,42,44,48} \mathrm{Ca}^{+}$isotopes with an accuracy of $\mathcal{O}(100)$ $\mathrm{kHz}$ corresponding to a fractional accuracy on the isotope shifts in the $10^{-5}-10^{-4}$ range [14]. In principle, far better accuracy can be achieved on narrow-optical transitions [15] such as the two $4 \mathrm{~s}-3 \mathrm{~d}$ quadrupole transitions. While the $4 \mathrm{~s}^{2} \mathrm{~S}_{1 / 2} \leftrightarrow 3 \mathrm{~d}^{2} \mathrm{D}_{5 / 2}(729-\mathrm{nm})$ transition has been measured at the $\mathrm{Hz}$ level in ${ }^{40} \mathrm{Ca}^{+}$[16], measurement of the $4 \mathrm{~s}^{2} \mathrm{~S}_{1 / 2} \leftrightarrow 3 \mathrm{~d}{ }^{2} \mathrm{D}_{3 / 2}$ (732-nm) transition at the same level is more challenging since the electronshelving technique [17] cannot directly be used for state detection.

In this letter, we report isotope shift measurements of the $3 \mathrm{~d}^{2} \mathrm{D}_{3 / 2}-3 \mathrm{~d}{ }^{2} \mathrm{D}_{5 / 2}$ interval (i.e. the D-finestructure splitting isotope shift (DSIS)) on all five stable even isotopes of ${ }^{A} \mathrm{Ca}^{+}$(with $\mathrm{A}=40$ as the reference isotope) using direct frequency-comb Raman spectroscopy [18]. Combining these with isotope shift measurements of the $4 \mathrm{~s}^{2} \mathrm{~S}_{1 / 2} \leftrightarrow 3 \mathrm{~d}^{2} \mathrm{D}_{5 / 2}$ transition (729-IS), we deduce the isotope shift of the $4 \mathrm{~s}^{2} \mathrm{~S}_{1 / 2} \leftrightarrow 3 \mathrm{~d}{ }^{2} \mathrm{D}_{3 / 2}$ transition (732-IS). This leads to a King plot analysis with unprecedented sensitivity to NP bosons coupling to both electrons and neutrons. In addition, the analysis yields a field shift ratio of the 729-nm and 732-nm transitions with an unprecedented fractional accuracy of $2 \times 10^{-7}$. We achieve an absolute accuracy on the DSIS at the 20 $\mathrm{Hz}$ level corresponding to a fractional accuracy in the 


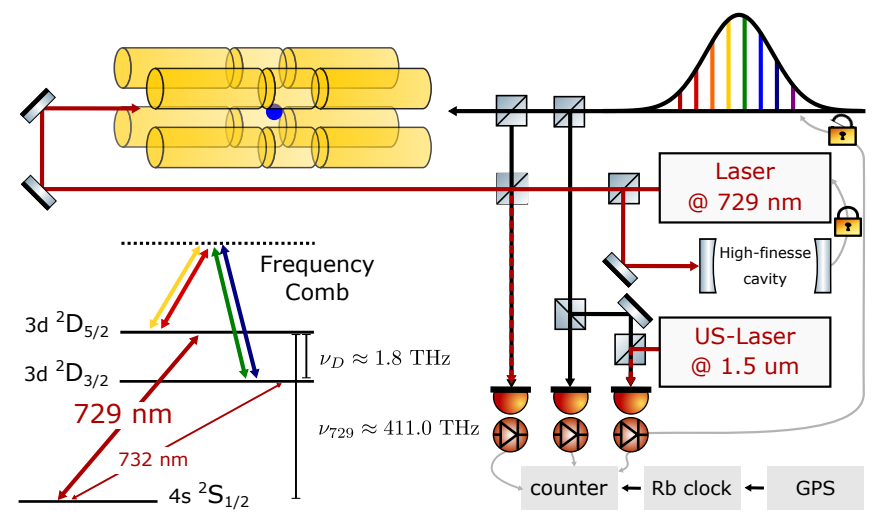

FIG. 1. Schematic of the experimental setup and the relevant electronic levels of the $\mathrm{Ca}^{+}$ion. The isotope shift of the 732-nm transition can be deduced from the isotope shifts of the 729-nm transition and of the D-fine-structure splitting. The D-fine-structure splitting is measured successively on the different calcium isotopes by direct frequency-comb Raman spectroscopy [18]. The transition frequency is deduced from the measurement of the comb repetition rate on a frequency counter referenced to a GPS-disciplined rubidium standard. The 729-nm laser used to probe the $4 \mathrm{~s}^{2} \mathrm{~S}_{1 / 2} \leftrightarrow 3 \mathrm{~d}^{2} \mathrm{D}_{5 / 2}$ transition is locked to an ultra-stable high-finesse cavity providing a short-term linewidth $<1 \mathrm{kHz}$. The absolute laser frequency is deduced from a measurement on the frequency counter of the beating between the laser and the frequency comb with the latter locked to an ultra-stable laser at $1.5 \mu \mathrm{m}$.

$10^{-6}$ range, and on the 729 -IS at the $2 \mathrm{kHz}$ level corresponding to a fractional accuracy in the $10^{-7}$ range. We show that, with respect to bounds on NP bosons, our measurements are in fact equally precise as measuring the isotope shift of the two $4 \mathrm{~s}-3 \mathrm{~d}$ transitions with the same $20 \mathrm{~Hz}$ level accuracy, since the limiting fractional accuracy is the DSIS measurement. In particular, the King plot analysis is not improved through combination with recent 729-IS measurements at the $10^{-9}$ level by Knollmann et al. [1]. It is neither limited by the 729-IS involving the isotope ${ }^{46} \mathrm{Ca}^{+}$that was not measured in Ref. [1]. However, the combined data yield isotope shifts of the 732-nm transition with fractional accuracy below the $10^{-8}$ level.

The splitting isotope shift of the $3 \mathrm{~d}^{2} \mathrm{D}_{3 / 2}$ and $3 \mathrm{~d}^{2} \mathrm{D}_{5 / 2}$ states $\delta \nu_{\text {DSIS }}^{A, 40}$ was measured using direct frequency-comb Raman spectroscopy, as described in details in [18]. In brief, a single $\mathrm{Ca}^{+}$isotope is loaded into a linear Paul trap via isotope-selective photoionization in a neutral calcium beam $[19,20]$. An external magnetic field of $6.500(3) \mathrm{G}$ lifts the Zeeman degeneracy of the involved electronic energy levels by a few $\mathrm{MHz}$, allowing for Zeeman-resolved spectroscopy of the $\mathrm{D}_{3 / 2}-\mathrm{D}_{5 / 2}$ interval. The experimental cycle is initialized by Doppler cooling, followed by sideband cooling and finally optical pumping of the $\mathrm{Ca}^{+}$ion into one of its $\left|4 s^{2} S_{1 / 2}, m_{j}= \pm 1 / 2\right\rangle$ states. Next, the ion is prepared to the $\mid D_{5 / 2}, m_{j}=$ $\pm 1 / 2\rangle$ state using rapid adiabatic passage (RAP) [21,22]. Finally, direct frequency-comb Raman spectroscopy of the two $\left|D_{5 / 2}, m_{j}= \pm 1 / 2\right\rangle \leftrightarrow\left|D_{3 / 2}, m_{j}^{\prime}= \pm 1 / 2\right\rangle$ symmetric transitions is carried out [18]. The state of the ion is read out by the electron-shelving technique [17]. The first-order differential Zeeman shift induced by the static magnetic field is canceled by averaging the two transition frequencies. The differential AC-Stark shift induced by the frequency comb is reduced by taking advantage of the existence of a "magic polarization" [18], and the unshifted transition frequency is obtained by extrapolating the measured frequencies to zero light intensity. The measured absolute D-splitting isotope shifts $\delta \nu_{\mathrm{DSIS}}^{A, 40}$ corrected for systematic effects (i.e. second-order Zeeman shift and electric-quadrupole shift mainly [18]) are presented table I. The achieved relative accuracy ranges from 2 to $7 \times 10^{-6}$.

The $4 \mathrm{~s}{ }^{2} \mathrm{~S}_{1 / 2} \leftrightarrow 3 \mathrm{~d}{ }^{2} \mathrm{D}_{5 / 2}$ transition near $729 \mathrm{~nm}$ was measured by Rabi spectroscopy [23]. In an experimental sequence similar to the one described above, after the optical pumping stage, the two $\left|S_{1 / 2}, \pm 1 / 2\right\rangle \leftrightarrow\left|D_{5 / 2}, \mp 3 / 2\right\rangle$ transitions are probed consecutively with $\pi$ pulses. The interrogation laser is locked to an ultra-stable high-finesse cavity providing a sub-kHz linewidth at short term (see figure 1). The absolute laser frequency is measured by beating this laser with one tooth of the frequency comb with the latter locked to an acetylene-stabilized ultrastable fiber laser (Stabiخaser from Denmark's National Metrology Institute [24, 25]). The differential first-order Zeeman shift is once again canceled by averaging the two transition frequencies. These measurements are limited by the relative inaccuracy of our GPS-disciplined rubidium standard which was measured against the Stabi入aser to be $5 \times 10^{-12}$. This corresponds to a $2 \mathrm{kHz}$ accuracy on the $\mathrm{S}_{1 / 2}-\mathrm{D}_{5 / 2}$ transition and a relative accuracy on $\delta \nu_{729}^{A, 40}$ ranging from 2 to $7 \times 10^{-7}$. The deduced isotope shifts $\delta \nu_{729}^{A, 40}$ are given table I together with part-perbillion measurements of $\delta \nu_{729}^{42,44,48-40}$ reported by Knollmann et al. [1]. Combined with our DSIS measurements, the data of Ref. [1] are further used to calculate the isotope shifts of the $4 \mathrm{~s}^{2} \mathrm{~S}_{1 / 2} \leftrightarrow 3 \mathrm{~d}^{2} \mathrm{D}_{3 / 2}$ transition near $732 \mathrm{~nm}$ with a fractional accuracy better than $10^{-8}$, as also presented in table I.

The two leading contributions to the isotope shift in atomic transition frequencies are the mass shift (MS) and the field shift (FS) [26]. The MS originates from the difference of the nuclear mass which leads to differences in the nuclear recoil energy. The FS originates from the change in the effective nuclear charge radius, which leads to different electronic potentials near the origin. With these two contributions, the isotope shift of a transition $i$ between isotope $A$ and $A^{\prime}$ can be written to leading order as:

$\delta \nu_{i}^{A A^{\prime}} \equiv \nu_{i}^{A}-\nu_{i}^{A^{\prime}}=\delta \nu_{i, \mathrm{MS}}^{A A^{\prime}}+\delta \nu_{i, \mathrm{FS}}^{A A^{\prime}}=\frac{K_{i}}{\mu}+F_{i} \delta\left\langle r_{c}^{2}\right\rangle^{A A^{\prime}}$

where $K_{i}$ and $F_{i}$ are the mass and field shift constants respectively, $\delta\left\langle r_{c}^{2}\right\rangle^{A A^{\prime}}=\left\langle r_{c}^{2}\right\rangle^{A}-\left\langle r_{c}^{2}\right\rangle^{A^{\prime}}$ is the difference of the mean squared nuclear charge radii, and $\mu$ is the 
TABLE I. Isotope shifts relative to ${ }^{40} \mathrm{Ca}^{+}$in $\mathrm{MHz}$ and their 1 standard deviation $\sigma$ uncertainties.

\begin{tabular}{|c|c|c|}
\hline $\mathrm{A} \quad \delta \nu_{\text {DSIS }}^{A, 40}$ & $\delta \nu_{729}^{A, 40}$ & $\delta \nu_{732}^{A, 40 \mathrm{a}}$ \\
\hline \multirow[t]{2}{*}{$42-3.519896(24)$} & $2771.873(2)$ & $2775.393(2)$ \\
\hline & $2771.8724676(76)^{\mathrm{b}}$ & $2775.392363(25)^{\mathrm{c}}$ \\
\hline \multirow[t]{2}{*}{$44-6.792470(22)$} & $5340.888(2)$ & $5347.680(2)$ \\
\hline & $5340.8873946(78)^{\mathrm{b}}$ & $5347.679865(23)^{\mathrm{c}}$ \\
\hline $46-9.901524(21)$ & $7768.401(2)$ & $7778.302(2)$ \\
\hline \multirow[t]{2}{*}{$48-12.746 \quad 610(27)$} & $9990.383(2)$ & $10003.130(2)$ \\
\hline & $9990.3818700(63)^{\mathrm{b}}$ & $10003.128480(28)^{\mathrm{C}}$ \\
\hline
\end{tabular}

reduced mass given by [27]

$$
\mu=\mu^{A A^{\prime}}=\frac{m_{A^{\prime}}\left(m_{A}+m_{e}\right)}{m_{A}-m_{A^{\prime}}}
$$

where $m_{e}$ is the electron mass, and $m_{A}$ and $m_{A^{\prime}}$ are the masses of the nuclei of the two isotopes respectively. The nuclear masses can be deduced from the precisely determined masses of the neutral atomic calcium isotopes [28], the total mass of the electrons, and the sum of the electrons binding energies $E_{n}^{\mathrm{b}}$ :

$$
m_{A}=m_{A, \text { neutral atom }}-20 m_{e}+\sum_{n=1}^{20} E_{n}^{\mathrm{b}}
$$

where the electron binding energies have been extracted from the NIST database [29]. If the isotope shifts are measured for more than one transition, the equation 1 allows one to eliminate the typically poorly known $\delta\left\langle r_{c}^{2}\right\rangle^{A A^{\prime}}$ and to write the so-called King relation [8]:

$$
\mu \delta \nu_{i}^{A A^{\prime}}=K_{i}-\frac{F_{i}}{F_{j}} K_{j}+\frac{F_{i}}{F_{j}} \mu \delta \nu_{j}^{A A^{\prime}}
$$

which, to leading order within the SM, is a linear relation between the modified isotope shifts $\mu \delta \nu_{i}^{A A^{\prime}}$ and $\mu \delta \nu_{j}^{A A^{\prime}}$ of the two transitions $i, j$. A NP interaction mediated by a boson $\phi$ of spin $s$ with coupling strengths $y_{e}$ and $y_{n}$ to electrons and neutrons, respectively, modifies the isotope shift predictions of Eq. 1 as

$$
\delta \nu_{i}^{A A^{\prime}}=\frac{K_{i}}{\mu}+F_{i} \delta\left\langle r_{c}^{2}\right\rangle^{A A^{\prime}}+(-1)^{s} \frac{\hbar c}{4 \pi} \frac{y_{e} y_{n}}{\hbar c} X_{i} \gamma^{A A^{\prime}}
$$

where the electronic NP coefficient $X_{i}$ characterizes the overlap of the wave-functions of the lower and upper states of transition $i$ with the potential mediated by the boson, independent of the isotopes, and $\gamma^{A A^{\prime}}$ depends on the isotopes only, independent of the transition. If $\phi$ couples linearly to the nucleus, then $\gamma^{A A^{\prime}}=A-A^{\prime}$. As a consequence, the King relation in Eq. 4 is in this case not linear anymore. Therefore, searching for non-linearities of the corresponding King plot provides sensitivity to a NP interaction mediated by such a boson.

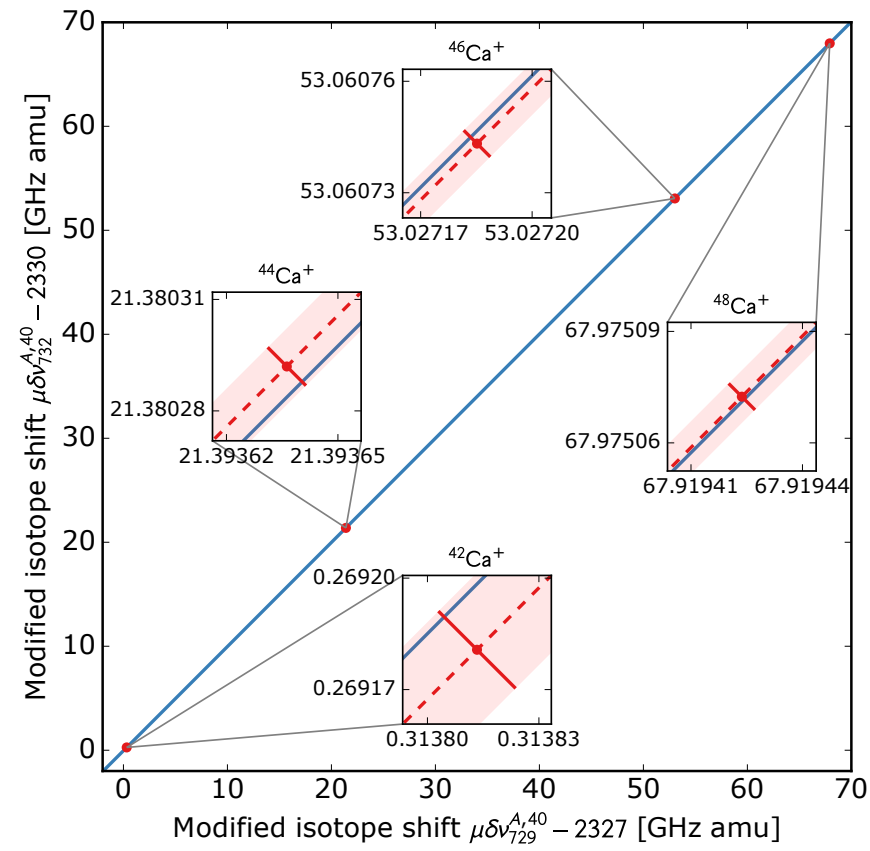

FIG. 2. Two-dimensional King plot of the 732-nm and 729$\mathrm{nm}$ transitions. The line is a fit to our data using a weighted orthogonal distance regression. The extracted fit parameters are given in the text. We point out that the isotope shift of the 732-nm transition is deduced from measurements of the isotope shift of the $729-\mathrm{nm}$ transition $\delta \nu_{729}^{A, 40}$ and of the D-splitting isotope shift $\delta \nu_{\text {DSIS }}^{A, 40}$. Hence, the measurement accuracy on $\delta \nu_{729}^{A, 40}\left(\delta \nu_{\text {DSIS }}^{A, 40}\right)$ translates into an error bar parallel (perpendicular) to the fitted line, emphasizing that the analysis is limited by the achieved fractional accuracy on $\delta \nu_{\text {DSIS }}^{A, 40}$.

The King plot of the modified isotope shift of the 732$\mathrm{nm}$ transition against the modified isotope shift of the 729-nm transition, using our experimental data only, is shown figure 2 . The blue line is a linear fit of the data using the King relation Eq. 4 and a Weighted Orthogonal Distance Regression [30]. We emphasize that $\delta \nu_{732}^{A, 40}$ is deduced from measurements of $\delta \nu_{729}^{A, 40}$ and $\delta \nu_{\text {DSIS }}^{A, 40}$, and that $\delta \nu_{729}^{A, 40} \gg \delta \nu_{\mathrm{DSIS}}^{A, 40}$. Consequently, the measurement uncertainties on $\delta \nu_{729}^{A, 40}$ and $\delta \nu_{\text {DSIS }}^{A, 40}$ translate into error bars essentially parallel and perpendicular to the fitted line, illustrating that the analysis is limited nearly exclusively by the achieved accuracy on $\delta \nu_{\text {DSIS }}^{A, 40}$. In fact, as long as the fractional accuracy on $\delta \nu_{729}^{A, 40}$ is smaller than the fractional accuracy on $\delta \nu_{\text {DSIS }}^{A, 40}$, measuring the DSIS at, e.g., the $20 \mathrm{~Hz}$ level is equivalent to measuring both the 729-IS and the 732-IS with the same $20 \mathrm{~Hz}$ accuracy. This is a consequence of the King plot analysis being sensitive to the difference of isotope shifts of the $D_{3 / 2}$ and $\mathrm{D}_{5 / 2}$ states, and this demonstrates the potential of measuring the DSIS directly using direct frequency-comb Raman spectroscopy.

The reduced $\chi^{2}$ of the fit is 0.89 and the King plot is thus linear within our measurement uncertainty. The non-linearity (defined in the Supplemental Material [31]) 
is $1.26 \sigma$. This allows for translating our measurement uncertainty into a constraint on the coupling strength of a hypothetical boson $\phi$. For a Yukawa potential $V_{\mathrm{NP}}=(-1)^{s}(A-Z) \frac{\hbar c}{4 \pi} \frac{y_{e} y_{n}}{\hbar c} \frac{e^{-r m_{\phi} c / \hbar}}{r}$, where $Z$ is the number of protons, we calculate the electronic NP coefficients $X_{i}$ using Brueckner orbitals and including relativistic random phase approximation corrections to the operator (see [31]). By constraining the nonlinear term from data (see $[9,31]$ ) and using the theory calculation of $X_{i}$, we evaluate the bounds on $y_{e} y_{n}$ as a function of the new mediator's mass $m_{\phi}$ which are shown in Fig. 3. The red solid curve corresponds to the bound using our experimental data only, yielding $y_{e} y_{n} / \hbar c<6.9 \cdot 10^{-11}$ at the $2 \sigma$ level in the mass-less limit $\left(m_{\phi}=1 \mathrm{eV}\right)$. The combination of the 729-IS measurements of Ref. [1] with our measurements of the DSIS and of $\delta \nu_{729}^{40,46}$, however, does not improve the bound despite the thousand times better accuracy on $\delta \nu_{729}^{42,44,48-40}$, confirming that the accuracy on the DSIS is the limiting one (as long as $\left.\sigma_{S-D_{5 / 2}} \cdot F_{\mathrm{DSIS}} / F_{S-D_{5 / 2}}<\sigma_{\mathrm{DSIS}}\right)$ and illustrating the potential of measuring the DSIS directly. The combined bound coincides with the bound using purely our data (up to a relative difference of $1 \%$ ) and is therefore not displayed. The black curve corresponds to the previous best bound set by measurements of the isotope shift of the two $\mathrm{S}_{1 / 2}-\mathrm{P}_{1 / 2}$ and $\mathrm{D}_{3 / 2}-\mathrm{P}_{1 / 2}$ dipole-allowed transitions by Gebert et al. [14] limiting $y_{e} y_{n} / \hbar c<2 \cdot 10^{-9}$ for $m_{\phi}=0$. We note that despite the hundred times better relative accuracy on the two $4 \mathrm{~s}-3 \mathrm{~d}$ transition isotope shifts achieved in this work, the bounds on $y_{e} y_{n}$ are improved by less than a factor 100. This is because the electronic configurations of the $\mathrm{D}_{3 / 2}$ and $\mathrm{D}_{5 / 2}$ states are more similar than the ones of the relevant $S_{1 / 2}$ and $\mathrm{D}_{3 / 2}$ states of Ref. [14]. More stringent bounds could be placed by constraining King plot non-linearities with heavier elements provided that one can correct for the non-linearities already predicted at higher order within the SM [32]. Two promising elements are $\mathrm{Ba}^{+}$or $\mathrm{Yb}^{+}$ which both have five spin-0 isotopes and D-splittings of 24 and $42 \mathrm{THz}$, respectively. The projected constraints imposed by measuring the DSIS at the $20 \mathrm{~Hz}$ level and the $\mathrm{S}_{1 / 2}-\mathrm{D}_{5 / 2}$ transition isotope shifts at the $\mathrm{kHz}$ level in $\mathrm{Ba}^{+}$ (green, dashed) and $\mathrm{Yb}^{+}$(dark blue, dashed) are also plotted in Fig. 3 (see [31]). Furthermore, we estimate the sensitivity of $\mathrm{Ca}^{+}, \mathrm{Ba}^{+}$and $\mathrm{Yb}^{+}$for measurements of the DSIS with $10 \mathrm{mHz}$ accuracy and of the $\mathrm{S}_{1 / 2}-\mathrm{D}_{5 / 2}$ transition isotope shifts with $\sim \mathrm{Hz}$ accuracy, under the condition that the uncertainty is limited by the isotope shift measurements and not by the uncertainty on the masses. The current constraints on $y_{e} y_{n}$ from King plot analyses, included the new bound derived in this work, are weaker than the astrophysical bound from star cooling of globular clusters [33-37] for $m_{\phi} \lesssim 0.3 \mathrm{MeV} / c^{2}$ and weaker than constraint on $y_{e}$ from the magnetic dipole moment $(g-2)$ of the electron $[38,39]$ combined with the constraint on $y_{n}$ from neutron scattering [40-43]. In contrast, the improved accuracy of the DSIS and $\mathrm{S}_{1 / 2}-\mathrm{D}_{5 / 2}$ measurements have the potential to probe so far uncon-

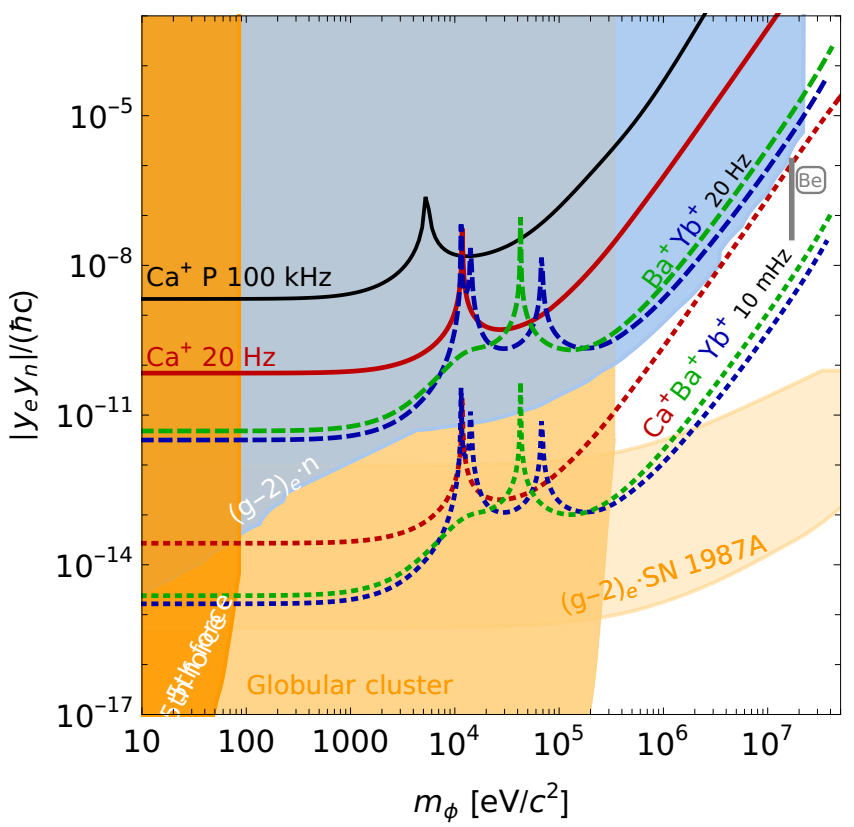

FIG. 3. Current and projected constraints $(2 \sigma)$ on the coupling strength $y_{e} y_{n}$ of electrons and neutrons to a new boson $\phi$ of mass $m_{\phi}$. Existing bound [9] from measurements of the $\mathrm{S}_{1 / 2}-\mathrm{P}_{1 / 2}$ and $\mathrm{D}_{3 / 2}-\mathrm{P}_{1 / 2}$ transition isotope shifts in $\mathrm{Ca}^{+}$with an accuracy of $\mathcal{O}(100 \mathrm{kHz})$ [14] (black, labelled as P). Constraint imposed by this work (red, solid), limited by the $\sim$ $20 \mathrm{~Hz}$ measurement uncertainty of the DSIS. Projection for a $10 \mathrm{mHz}$ uncertainty on the DSIS (red, dotted). Projected constraints from measurements in $\mathrm{Ba}^{+}$(DSIS at $20 \mathrm{~Hz}$ level, green dashed; $10 \mathrm{mHz}$, green dotted) and in $\mathrm{Yb}^{+}$isotopes (dark blue, also for $20 \mathrm{~Hz}$ and $10 \mathrm{mHz}$ ) (for details see [31]). The curves end at $m_{\phi}$ corresonding to the corresponding inverse nuclear radii. For comparison, constraints from other experiments are shown as shaded areas [9]: fifth force [44, 45] (dark orange), $(g-2)_{e}$ measurements $[38,39]$ combined with neutron scattering data [40-43] (light blue), or SN 1987A (light orange), and star cooling in globular clusters [33-37] (orange). The gray bar represents the range of $y_{e} y_{n}$ needed to explain the Be anomaly [9-13].

strained parameter space for $m_{\phi} \gtrsim 0.3 \mathrm{MeV} / c^{2}$ and in particular the range of $y_{e} y_{n}$ at $m_{\phi}=17 \mathrm{MeV} / c^{2}$ needed to explain the Be anomaly.

Finally, considering the case without a NP contribution, the fit parameters of the King plot analysis are $K_{21}=K_{732}-F_{732} / F_{729} K_{729}=-0.4961(5) \mathrm{GHz} . \mathrm{amu}$ and $F_{21}=F_{732} / F_{729}=1.00148305(20)$. Notably, we extract the ratio of the field shift constants with a relative accuracy of $2 \times 10^{-7}$ and the obtained value matches well the theoretical value calculated using many-body perturbation theory (see [31]) $F_{21}^{\mathrm{MBPT}}=1.0016$. We mention that this is also the case for the field shift ratio of the $\mathrm{S}_{1 / 2}-\mathrm{P}_{1 / 2}$ and $\mathrm{S}_{1 / 2}-\mathrm{P}_{3 / 2}$ transitions which isotope shifts were recently measured by Mller et al. [46], solving the field shift puzzle introduced with previous measurements made by Shi et al. [47]. Lastly, our data could be used to improve the accuracy on $\delta\left\langle r_{c}^{2}\right\rangle^{A A^{\prime}}$ for the even calcium 
isotopes considered here [48].

In summary, we have reported measurements of the D-fine-structure splitting isotope shift using direct frequency-comb Raman spectroscopy on all stable even isotopes of ${ }^{A} \mathrm{Ca}^{+}(\mathrm{A}=40,42,44,46$, and 48) with an accuracy of $\sim 20 \mathrm{~Hz}$. Combined with isotope shift measurements of the $4 \mathrm{~s}^{2} \mathrm{~S}_{1 / 2} \leftrightarrow 3 \mathrm{~d}^{2} \mathrm{D}_{5 / 2}$ transition at the $2 \mathrm{kHz}$ level, we performed a King plot analysis of the $4 \mathrm{~s}^{2} \mathrm{~S}_{1 / 2} \leftrightarrow 3 \mathrm{~d}^{2} \mathrm{D}_{5 / 2}$ and $4 \mathrm{~s}^{2} \mathrm{~S}_{1 / 2} \leftrightarrow 3 \mathrm{~d}^{2} \mathrm{D}_{3 / 2}$ transitions with unprecedented accuracy and extracted the field shift ratio with a fractional accuracy of $2 \times 10^{-7}$. Furthermore, the achieved uncertainty on the King plot linearity was used to improve isotope-shift-based bounds on the coupling strength of a New Physics boson to both electrons and neutrons. More stringent bounds could be placed by looking for King plot non-linearities with heavier elements, such as $\mathrm{Ba}^{+}$or $\mathrm{Yb}^{+}$applying direct frequencycomb Raman spectroscopy. Finally, NP interactions mediated by bosons with masses $m_{\phi} \gtrsim 0.3 \mathrm{MeV} / c^{2}$ and so far unconstrained by experiments, could be probed by measuring, with existing techniques for optical atomic clocks, the DSIS at the $10 \mathrm{mHz}$ level and one of the S-D isotope shifts at the $\sim \mathrm{Hz}$ level.

\section{ACKNOWLEDGMENTS}

We thank J. Hur, V. Vuletić, M. Schlaffer, R. Ozeri and P. O. Schmidt for interesting discussions. We acknowledge the Danish Center for Laser Infrastructure, LASERLAB.DK, established through the support of the Danish Ministry of Research and Education, for financial support and access to the frequency comb. We also acknowledge support from Innovation Fund Denmark, through the Quantum Innovation Center, Qubiz, for financial support and access to the Stabi入aser. C. S. acknowledges support from the European Commission through the Marie Curie Individual Fellowship COMAMOC (grant agreement no 795107) under Horizon 2020. S. M. and K. F. acknowledge support from the European Commission through the Marie Curie Initial Training Network COMIQ (grant agreement no 607491) under FP7. E. F. was supported during part of this work by the Minerva Foundation. J. C. B. is supported in this work by the Australian Research Council (DP190100974). M. D. acknowledges support from the European Commissions FET Open TEQ, the Villum Foundation and the Sapere Aude Initiative from the Independent Research Fund Denmark.
[1] F. W. Knollmann, A. N. Patel, and S. C. Doret, Physical Review A 100 (2019), 10.1103/physreva.100.022514.

[2] M. Mangano, arXiv preprint arXiv:2003.05976 (2020).

[3] D. BETTONI, S. BIANCO, F. BOSSI, M. CATANESI, A. CECCUCCI, F. CERVELLI, M. DELLORSO, U. DOSSELLI, F. FERRONI, and M. GRASSI, Physics Reports 434, 47 (2006).

[4] D. DeMille, J. M. Doyle, and A. O. Sushkov, Science 357, 990 (2017).

[5] M. Safronova, D. Budker, D. DeMille, D. F. J. Kimball, A. Derevianko, and C. W. Clark, Reviews of Modern Physics 90 (2018), 10.1103/revmodphys.90.025008.

[6] C. Delaunay, C. Frugiuele, E. Fuchs, and Y. Soreq, Physical Review D 96 (2017), 10.1103/physrevd.96.115002.

[7] C. Delaunay, R. Ozeri, G. Perez, and Y. Soreq, Physical Review D 96 (2017), 10.1103/physrevd.96.093001.

[8] W. H. King, Journal of the Optical Society of America 53, 638 (1963).

[9] J. C. Berengut, D. Budker, C. Delaunay, V. V. Flambaum, C. Frugiuele, E. Fuchs, C. Grojean, R. Harnik, R. Ozeri, G. Perez, and Y. Soreq, Physical Review Letters 120 (2018), 10.1103/physrevlett.120.091801.

[10] C. Frugiuele, E. Fuchs, G. Perez, and M. Schlaffer, Physical Review D 96 (2017), 10.1103/physrevd.96.015011.

[11] J. L. Feng, B. Fornal, I. Galon, S. Gardner, J. Smolinsky, T. M. P. Tait, and P. Tanedo, Phys. Rev. Lett. 117, 071803 (2016), arXiv:1604.07411 [hep-ph].

[12] J. L. Feng, B. Fornal, I. Galon, S. Gardner, J. Smolinsky, T. M. P. Tait, and P. Tanedo, Phys. Rev. D 95, 035017 (2017), arXiv:1608.03591 [hep-ph].

[13] A. Krasznahorkay, M. Csatlós, L. Csige, Z. Gácsi, J. Gulyás, M. Hunyadi, I. Kuti, B. Nyakó, L. Stuhl, J. Timár, T. Tornyi, Z. Vajta, T. Ketel, and A. Krasznahorkay, Physical Review Letters 116 (2016), 10.1103/physrevlett.116.042501.

[14] F. Gebert, Y. Wan, F. Wolf, C. N. Angstmann, J. C. Berengut, and P. O. Schmidt, Phys. Rev. Lett. 115, 053003 (2015).

[15] T. Manovitz, R. Shaniv, Y. Shapira, R. Ozeri, and N. Akerman, Physical Review Letters 123 (2019), 10.1103/physrevlett.123.203001.

[16] M. Chwalla, J. Benhelm, K. Kim, G. Kirchmair, T. Monz, M. Riebe, P. Schindler, A. Villar, W. Hänsel, C. Roos, et al., Physical review letters 102, 023002 (2009).

[17] H. G. Dehmelt, IEEE Transactions on Instrumentation and Measurement IM-31, 83 (1982).

[18] C. Solaro, S. Meyer, K. Fisher, M. DePalatis, and M. Drewsen, Physical Review Letters 120 (2018), 10.1103/physrevlett.120.253601.

[19] N. Kjærgaard, L. Hornekær, A. Thommesen, Z. Videsen, and M. Drewsen, Applied Physics B: Lasers and Optics 71, 207 (2000).

[20] A. Mortensen, J. J. T. Lindballe, I. S. Jensen, P. Staanum, D. Voigt, and M. Drewsen, Physical Review A 69, 042502 (2004).

[21] A. Turrin, Optics Communications 23, 220 (1977).

[22] C. Wunderlich, T. Hannemann, T. Körber, H. Häffner, C. Roos, W. Hänsel, R. Blatt, and F. Schmidt-Kaler, Journal of Modern Optics 54, 1541 (2007).

[23] I. I. Rabi, S. Millman, P. Kusch, and J. R. Zacharias, Phys. Rev. 55, 526 (1939).

[24] http://www.stabilaser.dk/.

[25] T. Talvard, P. G. Westergaard, M. V. DePalatis, N. F. Mortensen, M. Drewsen, B. Gøth, and J. Hald, Optics Express 25, 2259 (2017).

[26] W. R. Johnson, Atomic Structure Theory (Springer Berlin Heidelberg, 2007). 
[27] F. Kurth, T. Gudjons, B. Hilbert, T. Reisinger, G. Werth, and A. M. Mårtensson-Pendrill, Zeitschrift für Physik D Atoms, Molecules and Clusters 34, 227 (1995).

[28] M. Wang, G. Audi, F. Kondev, W. Huang, S. Naimi, and X. Xu, Chinese Physics C 41, 030003 (2017).

[29] A. Kramida, Y. Ralchenko, J. Reader, and NIST ASD Team, NIST Atomic Spectra Database (version 5.6.1) (2018), https://doi.org/10.18434/T4W30F.

[30] P. T. Boggs, R. H. Byrd, and R. B. Schnabel, SIAM Journal on Scientific and Statistical Computing 8, 1052 (1987).

[31] See Supplemental Material at [URL will be inserted by publisher] for details regarding the calculation of the bounds.

[32] V. V. Flambaum, A. J. Geddes, and A. V. Viatkina, Physical Review A 97 (2018), 10.1103/physreva.97.032510.

[33] W. M. Yao et al. (Particle Data Group), J. Phys. G33, 1 (2006).

[34] J. A. Grifols and E. Masso, Phys. Lett. B173, 237 (1986).

[35] J. A. Grifols, E. Masso, and S. Peris, Mod. Phys. Lett. A4, 311 (1989).

[36] J. Redondo and G. Raffelt, JCAP 1308, 034 (2013), arXiv:1305.2920 [hep-ph].

[37] E. Hardy and R. Lasenby, JHEP 02, 033 (2017), arXiv:1611.05852 [hep-ph].

[38] C. Patrignani et al. (Particle Data Group), Chin. Phys. C40, 100001 (2016).

[39] D. Hanneke, S. F. Hoogerheide, and G. Gabrielse, Physical Review A83, 052122 (2011), arXiv:1009.4831 [physics.atom-ph].

[40] R. Barbieri and T. E. O. Ericson, Phys. Lett. 57B, 270 (1975).
[41] H. Leeb and J. Schmiedmayer, Physical Review Letters 68, 1472 (1992).

[42] Yu. N. Pokotilovski, Phys. Atom. Nucl. 69, 924 (2006), arXiv:hep-ph/0601157 [hep-ph].

[43] V. V. Nesvizhevsky, G. Pignol, and K. V. Protasov, Physical Review D77, 034020 (2008), arXiv:0711.2298 [hep-ph].

[44] M. Bordag, U. Mohideen, and V. M. Mostepanenko, Phys. Rept. 353, 1 (2001), arXiv:quant-ph/0106045 [quant-ph].

[45] M. Bordag, G. L. Klimchitskaya, U. Mohideen, and V. M. Mostepanenko, Int. Ser. Monogr. Phys. 145, 1 (2009).

[46] P. Müller, K. König, P. Imgram, J. Krämer, and W. Nörtershäuser, To be published soon: "Collinear laser spectroscopy of $\mathrm{Ca}^{+}$: Solving the field-shift puzzle of the $4 \mathrm{~s}^{2} \mathrm{~S}_{1 / 2}-4 \mathrm{p}{ }^{2} \mathrm{P}_{1 / 2,3 / 2}$ transitions".

[47] C. Shi, F. Gebert, C. Gorges, S. Kaufmann, W. Nörtershäuser, B. K. Sahoo, A. Surzhykov, V. A. Yerokhin, J. C. Berengut, F. Wolf, J. C. Heip, and P. O. Schmidt, Applied Physics B 123, 2 (2016).

[48] A. Kramida, Atomic Data and Nuclear Data Tables , 101322 (2020).

[49] V. A. Dzuba, V. V. Flambaum, P. G. Silvestrov, and O. P. Sushkov, J. Phys. B 18, 597 (1985).

[50] E. V. Kahl and J. C. Berengut, Comp. Phys. Commun. 238, 232 (2019).

[51] I. Counts, J. Hur, D. P. A. Craik, H. Jeon, C. Leung, J. Berengut, A. Geddes, A. Kawasaki, W. Jhe, and V. Vuletić, (2020), arXiv:2004.11383 [physics.atom-ph].

[52] P. Villemoes, A. Arnesen, F. Heijkenskjold, and A. Wannstrom, 26, 4289 (1993).

[53] D. Hucul, J. E. Christensen, E. R. Hudson, and W. C. Campbell, Phys. Rev. Lett. 119, 100501 (2017).

\section{CALCULATION OF THE ELECTRONIC NP COEFFICIENTS}

Electronic coefficients of the Yukawa potential $X_{i}$ are calculated using the combination of Brueckner orbitals and random phase approximation (see [49] for details) implemented in the AMBiT code [50]. Briefly, we start with the Dirac-Fock method to generate core electrons and their potential. Valence and virtual orbitals are constructed by diagonalizing $B$ splines over the Dirac-Fock operator. To include the effects of core-valence correlations we use the $B$ spline basis orbitals to create an operator $\Sigma$ such that the second-order correlation correction to the energy of a valence orbital $|n\rangle$ is $\delta E_{n}^{(2)}=\langle n|\hat{\Sigma}| n\rangle$. This operator is then added to the Dirac-Fock operator $\hat{h}_{\mathrm{DF}}$ to create Brueckner orbitals defined by

$$
\left(\hat{h}_{\mathrm{DF}}+\hat{\Sigma}\right) \psi_{n}^{\mathrm{Br}}=E_{n}^{\mathrm{Br}} \psi_{n}^{\mathrm{Br}} .
$$

At lowest order, the electronic coefficients $X_{i}$ are simply the matrix elements $X_{i}=\left\langle\psi_{i}^{\mathrm{Br}}\left|V_{\mathrm{NP}}\right| \psi_{i}^{\mathrm{Br}}\right\rangle$. However the potential $V_{\mathrm{NP}}$ also polarises the core, and the resulting modification of the Dirac-Fock potential is a dominant effect for the valence $d$ orbitals, particularly in the limit of large mediator mass $m_{\phi}$. These core polarization effects are taken into account in the random phase approximation, which leads to a modified Dirac Fock potential $\delta V_{\mathrm{DF}}$ and electronic coefficients

$$
X_{i}=\left\langle\psi_{i}^{\mathrm{Br}}\left|V_{\mathrm{NP}}+\delta V_{\mathrm{DF}}\right| \psi_{i}^{\mathrm{Br}}\right\rangle .
$$

\section{GEOMETRIC PROJECTION METHOD FOR 4 ISOTOPE PAIRS}

In this work, we make - along with [51] - for the first time use of the New Physics implications of isotope shift measurements at high precision in five isotopes, i.e. four independent isotope pairs. While Ref. [9] developed the 
data-driven method for three isotope pairs and two transitions, and Ref. [10] performed a fit to measurements of either more isotopes (four isotope pairs of neutral $\mathrm{Yb}$ at $\mathrm{MHz}$ precision) or transitions (three transitions in $\mathrm{Ca}^{+}$without the $\left.A^{\prime}=46\right)$, here we take the four isotope pairs $A A^{\prime}$ with $A=40$ and $A^{\prime}=42,44,46,48$ into account by a geometric projection. The vectors of the measured isotope shifts

$$
\overrightarrow{\mu \delta \nu_{i}} \equiv\left\{\mu^{(1)} \delta \nu_{i}^{A A_{1}^{\prime}}, \mu^{(2)} \delta \nu_{i}^{A A_{2}^{\prime}}, \mu^{(3)} \delta \nu_{i}^{A A_{3}^{\prime}}, \mu^{(4)} \delta \nu_{i}^{A A_{4}^{\prime}}\right\}
$$

where $\mu^{A^{\prime}} \equiv \mu^{A A^{\prime}}$, are of dimension four. Hence a cross product as in Ref. [9] cannot be applied. Therefore, here we construct the measure of the non-linearity based on scalar products. We define the $4 \times 2$ matrix of isotope shift data as $D=\left(\overrightarrow{\mu \delta \nu_{1}}, \overrightarrow{\mu \delta \nu_{2}}\right)$. Then the projection of the (four-dimensional) modified mass shift vector $\mu=\{1,1,1,1\}$ onto the plane spanned by the isotope shift vectors $\overrightarrow{\mu \delta \nu_{1}}, \overrightarrow{\mu \delta \nu_{2}}$ is given by

$$
\vec{p}=\left[D \cdot\left(D^{T} D\right)^{-1} D^{T}\right] \vec{\mu}
$$

This allows us to calculate the volume of the parallelepiped spanned by the data vectors and the mass shift direction, which is proportional to the area spanned by the four points in the King plot, as

$$
V=|\vec{\mu}-\vec{p}| \sqrt{\left(\overrightarrow{\mu \delta \nu_{1}}\right)^{2}\left(\overrightarrow{\mu \delta \nu_{2}}\right)^{2}-\left(\overrightarrow{\mu \delta \nu_{1}} \cdot \overrightarrow{\mu \delta \nu_{2}}\right)}
$$

By error propagation of the measurements, we obtain the uncertainty $\sigma_{V}$ of $\mathrm{V}$. The significance of the non-linearity of a King plot, $\mathrm{NL}=V / \sigma_{V}$, is therefore determined purely from the measured isotope shifts and their uncertainties, independent of theory input. The non-linearity of the $\mathrm{Ca}^{+}$isotope shifts presented in this work is $V / \sigma_{V}=1.26$.

The bound plotted in Fig. 3 represents as $y_{e} y_{n}+2 \sigma_{y_{e} y_{n}}$ the approximate 95\% CL upper bound on

$$
y_{e} y_{n}\left(m_{\phi}\right)=\frac{V}{\left[X_{2}\left(m_{\phi}\right)-F_{21} X_{1}\left(m_{\phi}\right)\right] V_{\vec{h}}},
$$

where $V_{\vec{h}}$ is $V$ with $\overrightarrow{\mu \delta \nu_{2}}$ replaced by the NP direction $\vec{h}$ with $h_{A A^{\prime}}=\gamma_{A A^{\prime}} / \mu_{A A^{\prime}}$. For a linear coupling of $\phi$ to the nucleus, $\vec{h} \simeq-A \overrightarrow{A^{\prime}}$ amu. The uncertainty $\sigma_{y_{e} y_{n}}$ is obtained by error propagation of $V / V_{\vec{h}}$. For further details about the data-based non-linearity measure see Ref. [9].

\section{ESTIMATING NEAR-FUTURE SENSITIVITIES OF $\mathrm{CA}^{+}, \mathrm{BA}^{+} \mathrm{AND}_{\mathrm{YB}^{+}}$}

We estimate bounds from improved isotope-shift measurements in $\mathrm{Ca}^{+}, \mathrm{Ba}^{+}$and $\mathrm{Yb}^{+}$by two different methods. First, with Eq. (12) we use the analytic 'best-case' projection from Ref. [9] that does not require prior isotope shift measurements, but only depends on the absolute uncertainties of the isotope shifts, $\sigma_{1}, \sigma_{2}$. It neglects a possible alignment of the field shift with the NP and assumes perfect linearity. Therefore the bound is entirely determined by the uncertainty on $y_{e} y_{n}$

$$
\left[\sigma_{y_{e} y_{n}}\right]_{\text {proj }} \sim 4 \pi \frac{\sqrt{\sigma_{2}^{2}+\sigma_{1}^{2} F_{21}^{2}}}{\left(X_{2}-X_{1} F_{21}\right)} \frac{A}{\Delta A_{j}^{\min } \Delta A_{j}^{\max }} .
$$

As stated in [9], this projection will always indicate a stronger constraint than a bound from data at the same level of frequency uncertainties $\sigma_{i}$.

As the second method, in order to allow for a non-linearity of about $1 \sigma$ expected in any future measurement, we generate a mock data set with the targeted precision. We take $\mathrm{S}_{-} \mathrm{D}_{5 / 2}$ as the first transition $(i=1)$ from previous data (high precision is not required and at least two isotope pairs are sufficient) to predict the second transition $(i=2)$ $\mathrm{D}_{3 / 2}-\mathrm{D}_{5 / 2}$,

$$
\mu \delta \nu_{2}^{A A^{\prime}}=K_{21}+F_{21} \mu \delta \nu_{2}^{A A^{\prime}} \pm \delta \cdot \mu^{A A^{\prime}}
$$

where $\delta[\mathrm{Hz}]$ is a possible displacement from the straight line. $K_{21}$ and $F_{21}$ can be taken from available data or from theory. The generated data set $\left\{\left[\mu \delta \nu_{1}^{A A^{\prime}}\right]^{\text {data }},\left[\mu \delta \nu_{2}^{A A^{\prime}}\right]^{\text {mock }}\right\}$ and its uncertainty $\left\{\sigma_{1} \mu^{A A^{\prime}}, \sigma_{2} \mu^{A A^{\prime}}\right\}$ then yields via Eq. (11) a bound on $y_{e} y_{n}$. 
In the following paragraphs we provide element-specific information.

$\underline{\mathrm{Ca}^{+}}$We use $\mu \delta \nu_{S-D_{5 / 2}}$ and electronic coefficients from this paper.

$\underline{\mathrm{Ba}^{+}}$From Refs. $[52,53]$ we calculate the isotope shifts $\delta \nu_{S-D_{5 / 2}}^{138, A}, \delta \nu_{D_{3 / 2}-D_{5 / 2}}^{138, A}$ for $A=134,136$ and obtain $F_{21}^{\mathrm{Ba}}=$ -0.00491734 and $K_{21}=-17.454 \mathrm{GHz}$ amu to calculate the approximated isotope shifts of the pairs with $A=130,132$ assuming perfect linearity. The Ba masses are taken from [28]. In the evaluation of the bound on $y_{e} y_{n}$ according to Eq. (11), we use the theory value $F_{21}^{\mathrm{Ba}}=-0.0141905$ from our calculation with Eq. (7) in order to ensure convergence of $X_{2} / X_{1} \rightarrow F_{21}$ in the limit of large $m_{\phi}$.

$\underline{\mathrm{Yb}^{+}}$We use $\delta \nu_{S-D_{5 / 2}}^{A A^{\prime}}$ from the recent Counts et al. [51] with $A^{\prime}=A+2$ for the four pairs of $A=168,170,172,174$. Hence the elements of the NP vector $\vec{h}$ are given by $h_{n}=-A_{n} A_{n+1}$ amu $=-A_{n}\left(A_{n}+2\right)$ amu. From [51] we calculate $K_{21}=-65.8224 \mathrm{GHz}$ amu.

As two benchmarks we choose $\sigma_{2} \equiv \sigma_{D D}=20 \mathrm{~Hz}$ (the current level achieved for $\mathrm{Ca}^{+}$in this work) and $10 \mathrm{mHz}$ (achieved already for $\mathrm{Sr}^{+}[15]$ ) and set $\sigma_{S D}<\sigma_{D D} / F_{21}$ such that the bound will be determined by $\sigma_{D D}$ whereas an improvement of $\sigma_{S D}$ would not improve the bound significantly. We consider two options, see Tab. II: ( $i$ ) all points are on the straight line (i.e. the non-linearity vanishes by construction); (ii) we choose the displacement $\delta$ such that the significance of the resulting non-linearity is $V / \sigma_{V}=1$ where $V$ is the volume defined in Eq. 10 and $\sigma_{V}$ its uncertainty.

TABLE II. Upper limits $y_{e} y_{n}+2 \sigma_{y_{e} y_{n}}$ expected in the massless limit $\left(m_{\phi}=1 \mathrm{eV} / c^{2}\right)$ in two benchmarks. Comparison of limit from mock data from Eq. (13) with $V / \sigma_{V} \simeq 1 \sigma$ nonlinearity, exact linearity $(\delta=0)$ and from the simplified estimate of Eq. (12). $\mathrm{Ca}^{+}$with isotope pairs $\{42,44,46,48\}-40, \mathrm{Ba}^{+}$with $\{130,132,134,136\}-138$ and $\mathrm{Yb}^{+}$with neighboring pairs of $\{168,170,172,174,176\}$. The last lines contains the field shift ratio from theory and the sensitivity factor $X_{2}-F_{21} X_{1}$ for $m_{\phi}=1 \mathrm{eV} / c^{2}$. The example of $\mathrm{Ca}^{+}$with $\sigma_{2}=20 \mathrm{~Hz}$ is for comparison only, instead the bound from real data is plotted.

\begin{tabular}{l|lll}
\hline \hline & $\mathrm{Ca}^{+}$ & $\mathrm{Ba}^{+}$ & $\mathrm{Yb}^{+}$ \\
\hline$\delta, \sigma_{1}, \sigma_{2}[\mathrm{~Hz}]$ & $12,2000,20$ & $12,100,20$ & $10,100,20$ \\
$V / \sigma_{V}$ & 1.002 & 0.999 & 0.992 \\
$y_{e} y_{n} / \hbar c$ & $5.4 \cdot 10^{-11}$ & $4.8 \cdot 10^{-12}$ & $3.2 \cdot 10^{-12}$ \\
$y_{e} y_{n} / \hbar c, \delta=0$ & $4.3 \cdot 10^{-12}$ & $1.74 \cdot 10^{-12}$ & $3.9 \cdot 10^{-13}$ \\
$y_{e} y_{n} / \hbar c$, Eq. $(12)$ & $3.8 \cdot 10^{-12}$ & $1.72 \cdot 10^{-12}$ & $8.05 \cdot 10^{-13}$ \\
\hline$\delta, \sigma_{1}, \sigma_{2}[\mathrm{~Hz}]$ & $0.006,1,0.01$ & $0.006,0.1,0.01$ & $0.0055,0.1,0.01$ \\
$V / \sigma_{V}$ & 1.003 & 1.089 & 1.07 \\
$y_{e} y_{n} / \hbar c$ & $2.7 \cdot 10^{-14}$ & $2.4 \cdot 10^{-15}$ & $1.7 \cdot 10^{-15}$ \\
$y_{e} y_{n} / \hbar c, \delta=0$ & $2.2 \cdot 10^{-15}$ & $8.68 \cdot 10^{-16}$ & $2.0 \cdot 10^{-16}$ \\
$y_{e} y_{n} / \hbar c, \mathrm{Eq} .(12)$ & $1.9 \cdot 10^{-15}$ & $8.66 \cdot 10^{-16}$ & $4.1 \cdot 10^{-16}$ \\
\hline$F_{21}=F_{2} / F_{1}$ & -0.001795 & -0.01419 & -0.02152 \\
$X_{2}-F_{21} X 1[\mathrm{~Hz}]$ & $3.353 \cdot 10^{14}$ & $-2.527 \cdot 10^{15}$ & $-6.908 \cdot 10^{15}$ \\
\hline \hline
\end{tabular}

Tab. II shows that the mock data method with vanishing non-linearity reproduces the limit obtained from the analytic estimate where all points are assumed to be on a perfect line (apart from a difference in $\mathrm{Yb}^{+}$). Furthermore it shows that a non-linearity of $1 \sigma$ weakens the bound by up to an order of magnitude. In Fig. 3 we plot the bounds corresponding to a $1 \sigma$ non-linearity as a more realistic estimate.

For $\mathrm{Ca}^{+}$, we note that the mock data method with $\sigma_{S D}=2 \mathrm{kHz}, \sigma_{D D}=27 \mathrm{~Hz}$ and $\delta=19 \mathrm{~Hz}\left(V / \sigma_{V}=1.2\right)$ yields $y_{e} y_{n} / \hbar c<7.6 \cdot 10^{-11}$, i.e. it reproduces the bound from real data accurately. Furthermore, we compare the bound based on $\sigma_{D D}=27 \mathrm{~Hz}$ to the bound from measuring $\mathrm{S}_{-} \mathrm{D}_{3 / 2}$ and $\mathrm{S}-\mathrm{D}_{3 / 2}$ both at $20 \mathrm{~Hz}$ precision, obtaining for the example of zero non-linearity and $m_{\phi}=1 \mathrm{eV} / c^{2},\{5.3,5.1\} \cdot 10^{-12}$, respectively, hence confirming their equivalence. 